$\begin{array}{cl}\text { PRAMANA } & \text { (c) Indian Academy of Sciences } \\ \begin{array}{c}\text { journal of } \\ \text { physics }\end{array} & \begin{array}{l}\text { Vol. } 69, \text { No. } 6 \\ \text { December } 2007 \\ \text { pp. } 1165-1169\end{array}\end{array}$

\title{
The E166 experiment: Development of an undulator-based polarized positron source for the international linear collider
}

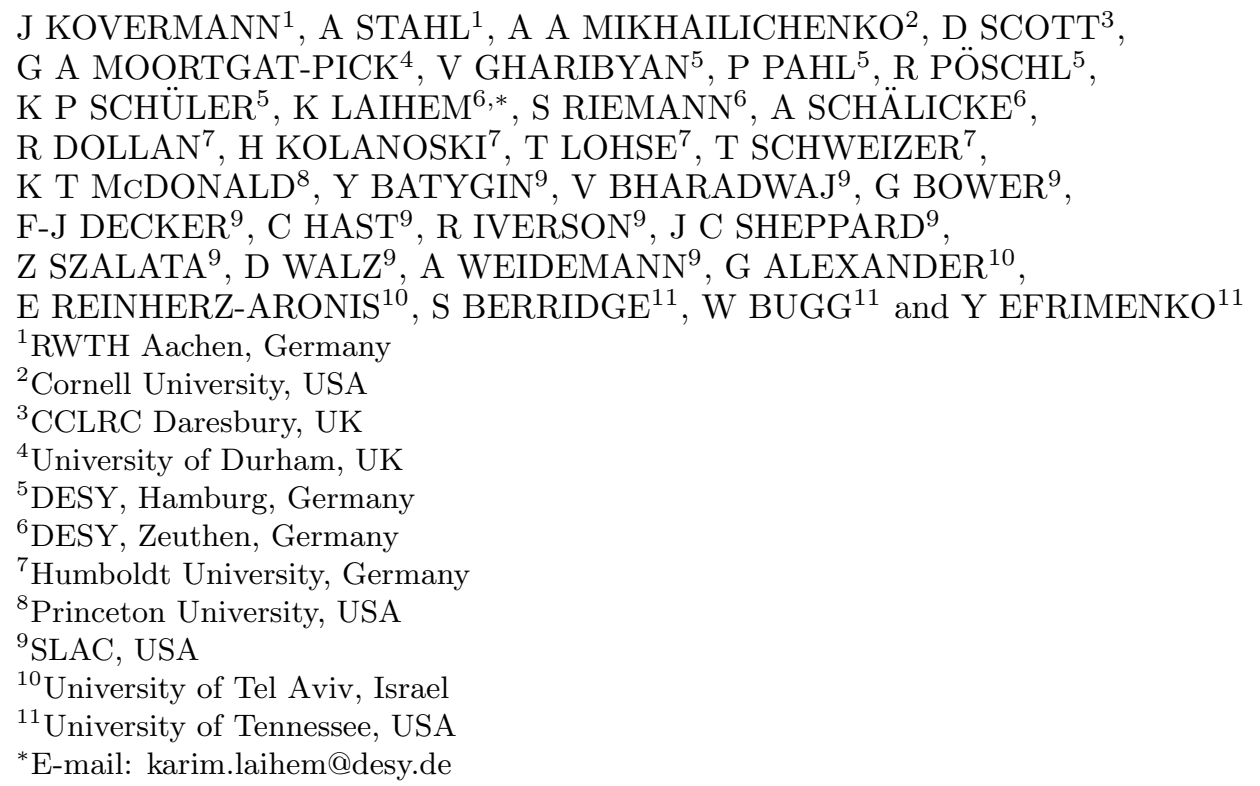

\begin{abstract}
A longitudinal polarized positron beam is foreseen for the international linear collider (ILC). A proof-of-principle experiment has been performed in the final focus test beam at SLAC to demonstrate the production of polarized positrons for implementation at the ILC. The E166 experiment uses a $1 \mathrm{~m}$ long helical undulator in a $46.6 \mathrm{GeV}$ electron beam to produce a few $\mathrm{MeV}$ photons with a high degree of circular polarization. These photons are then converted in a thin target to generate longitudinally polarized $e^{+}$and $e^{-}$. The positron polarization is measured using a Compton transmission polarimeter. The data analysis has shown asymmetries in the expected vicinity of $3.4 \%$ and $\sim 1 \%$ for photons and positrons respectively and the expected positron longitudinal polarization is covering a range from $50 \%$ to $90 \%$.
\end{abstract}

Keywords. International linear collider; helical undulator; polarized positron; Compton transmission polarimeter.

PACS No. 29.17 


\section{$J$ Kovermann et al}

\section{Introduction}

The full exploitation of the physics potential of an international linear collider (ILC) will require the development of polarized positron beams. Having both $e^{+}$and $e^{-}$ beams polarized will provide new insight into structures of couplings and thus give access to physics beyond the standard model [1]. The concept for a polarized positron source is based on circularly polarized photon sources. These photons are then converted to longitudinally polarized $e^{+}$and $e^{-}$pairs. While in an experiment at KEK [1a], Compton backscattering is used [2], the E166 experiment uses a helical undulator to produce polarized photons. An undulator-based positron source for the ILC has been proposed in $[3,4]$. The proposed scheme for an ILC positron source is illustrated in figure 1 . In this scheme, a $150 \mathrm{GeV}$ electron beam passes through a $120 \mathrm{~m}$ long helical undulator to produce an intense photon beam with a high degree of circular polarization. These photons are converted in a thin target to $e^{+} e^{-}$pairs. The polarized positrons are then collected, pre-accelerated to the damping ring and injected to the main linac. The E166 experiment is dedicated to test this concept of a helical-undulator-based polarized positron source for the ILC. The experiment was carried out at SLAC where it benefitted from the high quality electron beam in the final focus test beam (FFTB).

\section{The E166 experimental set-up and operation}

The $46.6 \mathrm{GeV}$ electron beam was sent through a $1 \mathrm{~m}$ long helical undulator with $0.89 \mathrm{~mm}$ aperture. When passing through the undulator, the electron beam is forced to radiate under a helical profile of the transverse magnetic field, providing circularly polarized photons (figure 2). The first harmonic energy cut-off depends on both the electron beam energy and the undulator period [4]. The undulator $K$-factor is about 0.19 which leads to a parabolic distribution for the first harmonic with a cut-off energy at $\sim 8 \mathrm{MeV}$.

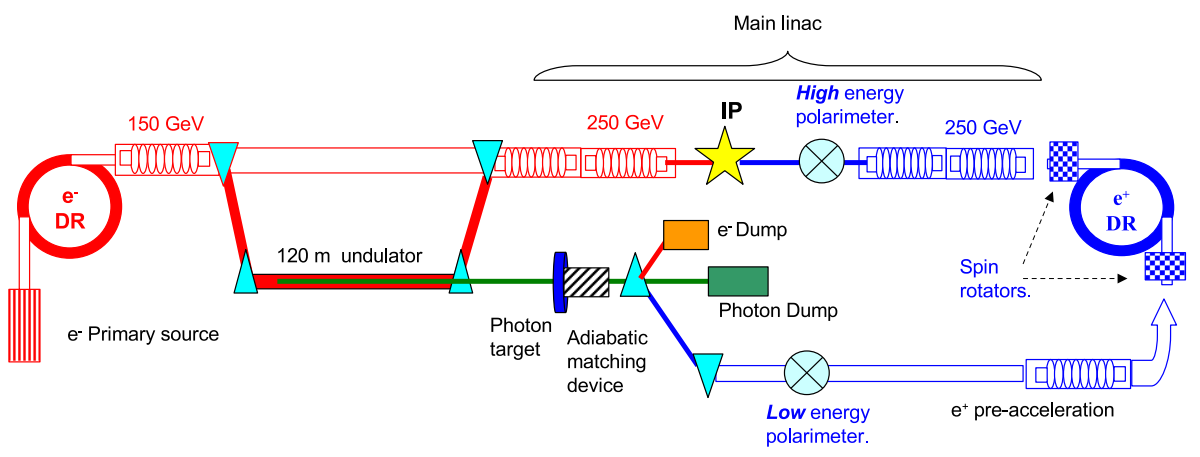

Figure 1. A sketch of the baseline design for the helical-undulator-based polarized positron source for the international linear collider (ILC). 
The E166 experiment

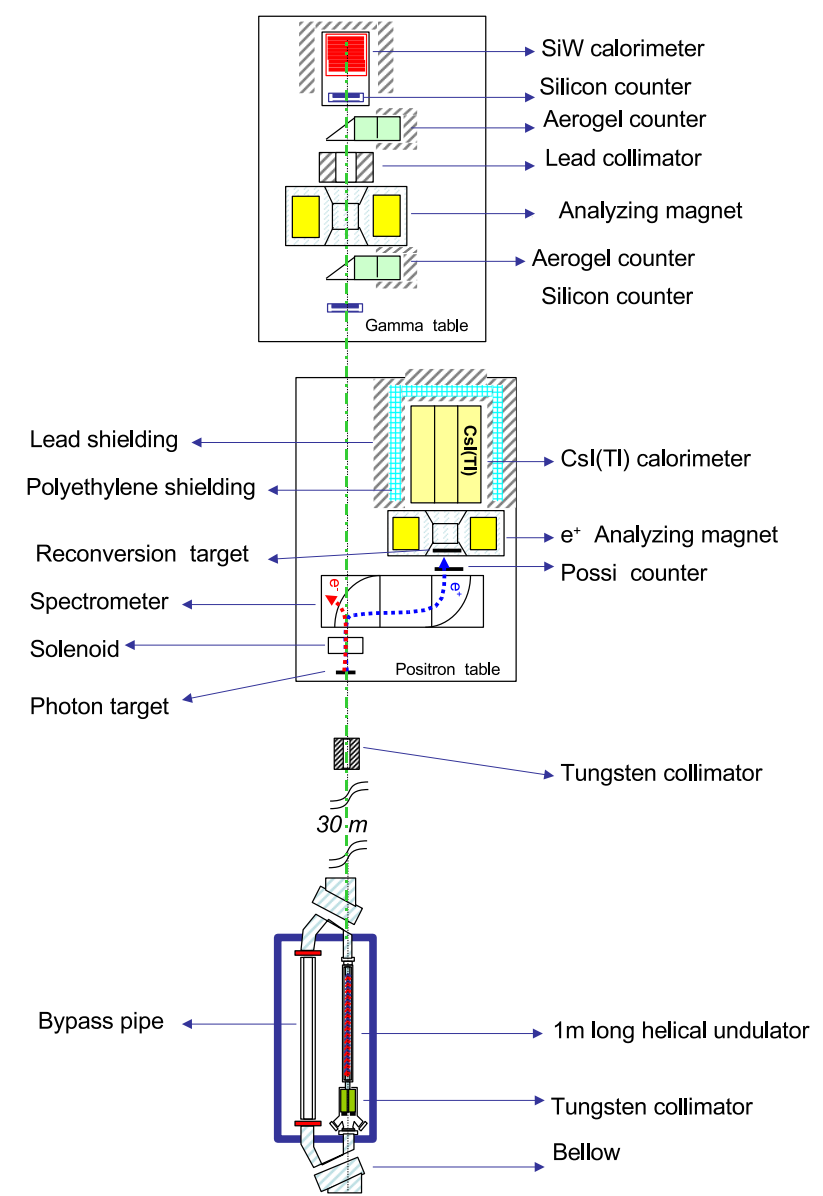

Figure 2. Synoptic scheme of the E166 experiment showing the most relevant components of the set-up at the FFTB.

In a $0.25 X_{0}$ tungsten target, located at about $\sim 30 \mathrm{~m}$ downstream the undulator, a very small fraction of the undulator photons is converted into $e^{+} e^{-}$pairs. In the pair production process the high energetic $e^{+}$inherit the polarization of the undulator photons [5]. The remaining photon beam passes then a set of counters and its polarization is measured by using a Compton transmission method [6] (figures $2,3)$.

The produced positrons are first separated from the electrons, filtered by their momentum in a magnetic spectrometer and re-converted into polarized photons via bremsstrahlung and annihilation processes. Here again, the produced photons inherit the positron polarization [5]. By using the same method as for the photon line, the photon polarization is measured and it translates back to the positron longitudinal polarization. To investigate the energy dependence of the positron longitudinal polarization [5], the E166 experiment has selected five positron momenta of 4.4, 5.3, 6.1,7.0 and $7.9 \mathrm{MeV}$. 


\section{$J$ Kovermann et al}
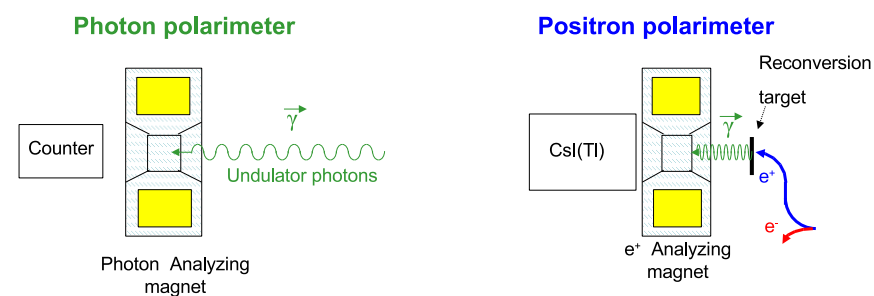

Figure 3. Basic concept of the Compton transmission polarimeter for the undulator photons and for positrons at the E166 experiment.

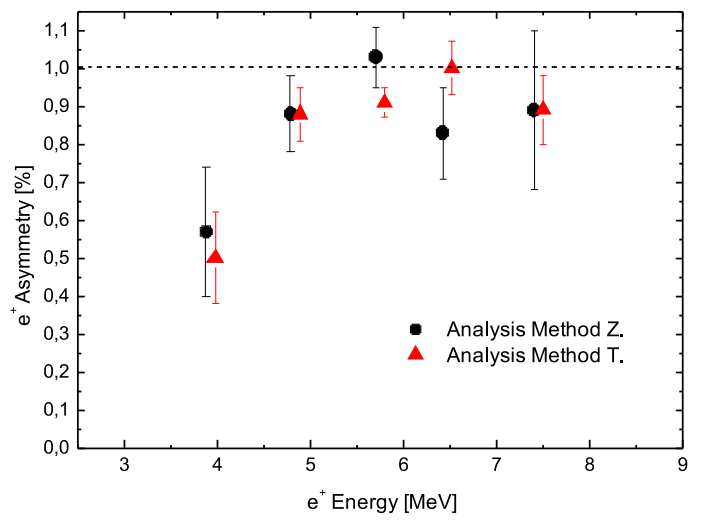

Figure 4. The positron asymmetry vs. energy. Methods Z and T stand for the Zeuthen and the Tel-Aviv analysis group respectively.

\section{Photon and positron polarimetry}

In the E166 experiment, both the circular polarization of photons and the longitudinal polarization of positrons are measured simultaneously using the Compton transmission polarimetry. This method is based on the spin-dependent Compton scattering of polarized photons with polarized target electrons in a magnetized iron. The Compton transmission method has the advantage that low-energy background photons are suppressed in the energy measurement. The iron is the best known ferromagnet which reaches $\sim 7 \%$ polarization at saturation. By flipping the iron magnetization, an asymmetry in the transmitted photon rate is observed. The asymmetry can be approximated by eq. (1).

$$
\delta=\frac{T_{+}-T_{-}}{T_{+}+T_{-}} \approx n L \sigma_{\mathrm{Pol}} P_{\gamma} P_{e}=A_{\gamma} P_{\gamma} P_{e} .
$$

$A_{\gamma}=n L \sigma_{\text {Pol }}$ is the analyzing power. $P_{\gamma}$ and $P_{e}$ stand for the photon and the target electron polarization. The photon polarization (proportional to the $e^{+}$polarization) can thus be inferred from the measured asymmetry if $A_{\gamma}$ and $P_{e}$ are known. 


\section{The E166 experiment}

The analyzing power (determined from Monte Carlo) is photon energy-dependent and must therefore be convoluted over the photon spectrum. In E166, the photon polarimeter consists of two main components: the analyzer magnet with a reversible magnetization of the iron core $(15 \mathrm{~cm}$ thick) and a $\mathrm{SiW}$ calorimeter for the energy and flux measurement (figure 3). The same concept is used for the positron polarization measurement. The positron has to be reconverted into bremsstrahlung/annihilation photons. The positron polarimeter consists of a reconversion target $\left(0.5 X_{0}\right.$ tungsten $)$, a magnetized iron $(7.5 \mathrm{~cm}$ thick) and a $\mathrm{CsI}(\mathrm{Tl})$ calorimeter (figures 2, 3). By reversing the iron magnetization, an asymmetry in the transmitted signal is recorded.

Two independent and different approaches for the data analysis show consistent results on the measured asymmetries (figure 4). The asymmetries are in the expected range for photons $\sim 3.4 \%$ and depending on the selected $e^{+}$energy, the positron asymmetry is in the range of $0.5 \%$ up to $1 \%$. E166 has also measured a $1.3 \%$ asymmetry in the electron signal. The results obtained for the positron asymmetries lead to preliminary longitudinal polarization values in the range of $50 \%$ up to $90 \%$. The analysis is ongoing. With this experimental confirmation, the helical undulator method became a preferred candidate for an ILC polarized positron source.

\section{References}

[1] G Moortgat-Pick and H M Steiner, EPJdirect C, vol. 1, C6, hep-ph/0106155 (2001)

[1a] At the ATF collaboration, the recent result of the Compton scheme shows longitudinal polarization of about $73 \%$ for both electrons and positrons [2]

[2] T Omori et al, hep-ex/0508026, KEK Preprint (2005)

[3] V E Balakin and A A Mikhailichenko, Preprint BINP 79-85 (1979)

[4] A A Mikhailichenko, Cornell CBN 02-10, SLAC LCC106 (Sept. 16, 2002)

[5] H Olsen and L C Maximon, Phys. Rev. 114, 887 (1959)

[6] M Woods et al, E166 proposal, SLAC LCC-0107 (Nov. 6, 2002) 